\title{
CONTEXTUAL CONTRADICTIONS TO THE SCALING -UP OF MOBILE HEALTH IN ARMED-CONFLICT SETTINGS OF NORTH-EASTERN NIGERIA: A CASE STUDY OF ALMANACH
}

\author{
Nakama David ${ }^{1}$, Muhammadou $\mathrm{Kah}^{2}$, Jennifer Tyndall ${ }^{3}$ and Olumide Longe ${ }^{4}$ \\ American University of Nigeria, Nigeria \\ ${ }^{1}+2348038013255$ \\ ${ }^{2}+2348149228426$ \\ ${ }^{3}+2348077092830$ \\ ${ }^{4}+2348160900893$
}

\begin{abstract}
Developing countries are undergoing an unprecedented growth on the adoption of ICT's. Mobile technologies, digital platforms and services for health care are gaining acceptance and use for detection and response for disease outbreaks, support of the healthcare value chains to facilitate improvements of public health care delivery and services. This study through a case study examines how m-Health platforms and services produce positive results in the context of Sub-Saharan Africa, specifically investigating the challenges of $m$-Health adoption and use in the Boko Haram settings in Adamawa State, North Eastern Part of Nigeria. Our study also explores the challenges that impede the successful scale-up of m-Health intervention in armed conflict settings of Adamawa State in the North Eastern region of the Federal Republic of Nigeria. We examined the use of ALMANACH in five local government areas (LGAs) namely; Yola south, Girei, Mubi-South, Mubi-North, and Maiha located in the armed-conflict (Boko Haram) settings of Adamawa State, Nigeria. Nineteen PHCs where ALMANACH has been deployed, primarily conducted in children clinics. A m-Health: "Algorithm for the Management of Acute Childhood Illnesses" (ALMANACH) was introduced as a clinical decision support tool into the child health system to aid the management of common acute illnesses in children between 2 months and 5 years. The ALMANACH is an electronic version of the integrated management of childhood illnesses (IMCI) running on mobile tablets. The lens of activity theory has guided the study to unearth the emergence of some developments in ALMANACH adoption and use in our study setting. Activity theory (AT) was used as the analytical lens because it is central for understanding challenges in adoption and use which can present in the form of contradictions. Thus, our findings suggest several challenges in the form of contradictions that can impede the successful scale-up of ALMANACH in peculiar settings of Boko Haram. Hence, a key contribution to the literature from our study is uncovering that contradictions are critical to achieving mHealth scale-up in our study setting.
\end{abstract}

\section{KEYWORDS}

mHealth, Healthcare Services, Armed-Conflict, ALMANACH, Contradictions

\section{INTRODUCTION}

Armed-conflict zones usually present some of the most precarious health systems with poor human capacity and significant governance challenges. There is also an acute shortage of human resources for health care service delivery in conflict and post-conflict settings due to the migration of large numbers of qualified health workers, as well as the destruction of educational and health facilities (Devkota \& van Teijlingen, 2010; Woodward et al., 2014) . These challenges have contributed greatly to the poor child health outcomes as well as the high child mortality rates especially in developing countries. In 2015, about 6 million children between age 0 and 5 died of curable and preventable illnesses in conflict-affected countries like Syria, Nigeria, and Niger (Asi \& Williams, 2018). In an attempt to augment these healthcare deficiencies, mobile health (mHealth) solutions have witnessed a high number of implementations in many disadvantaged regions of the world including conflict and post- conflict regions (Woodward et al., 2014). mHealth can have a resounding and 
positive effect on health care delivery in the most disadvantaged regions around the world (Chigona et al., 2012; Motamarri et al., 2014), if it is properly implemented and effectively scaled-up. Such is especially the case in the areas of reproductive, maternal and child health (Chigona et al., 2012). By leveraging on mobile technologies to improve child health outcomes, mHealth has experienced unprecedented growth in recent years with about 500 distinct pilot implementations globally, the majority of these developments have taken place in developing countries (Tomlinson et al., 2013). This approach has been found to overcome widespread health system barriers such as health professional shortages, reliance on untrained and/or informal providers, cost of service and transportation, and lack of sources of reliable information (WHO,2011). Regardless of the enthusiasm around mHealth and its great potentials in improving the health care landscape of developing countries (Motamarri et al., 2014; Schmied et al., 2010), the majority of mHealth interventions are faced with challenges which hampers the scale-up of such interventions (Bilandzic \& Venable, 2011; Chigona et al., 2012; Fanta \& Pretorius, 2018; Mangone et al., 2016).

Related studies have illuminated on mHealth scale-up with some wide range methods, outcome measures, and scope (Dehzad et al., 2014; Sanner et al., 2012). While these studies were successful in exploring the different measures of taking mHealth pilots to full scale-up across diverse settings, experts in the field have acknowledged the relative absence of a rigorous scholarly approach of how mHealth scale-up can be achieved (Tomlinson et al., 2013). There is also, the need to study the challenges of mHealth implementations in developing countries (Kruse et al., 2019). Thus, this study aims to explore the challenges that impede the successful scale-up of mHealth intervention in developing countries. Particularly, armed conflict settings. Such studies are scarce in literature. Hence, knowledge from this study will contribute to extant literature on mHealth implementations. The study may serve as a valuable tool for intervention donors and policymakers to consider when planning or designing imminent mHealth projects. Findings from this study will also strengthen the chances of long-term, sustainable scale-up of mHealth in armed conflict settings of developing countries. This study was conducted within a child health care program in the armed conflict settings (Boko Haram) of North-East (NE) Nigeria. A mHealth: "Algorithm for the Management of Acute Childhood Illnesses" (ALMANACH) was introduced as a clinical decision support tool into the child health system to aid the management of common acute illnesses in children between 2 months and 5 years. Activity theory (AT) was used as the analytical lens because it is central for understanding challenges in practice which can present in the form of contradictions (Shidende, 2014). With that understanding, the following research question is addressed: how can the challenges faced by mHealth systems in armed conflict settings affect its scale-up?

\section{THEORETICAL FOUNDATION}

Activity theory is a social-psychological theory that is based on the work of the Russian psychologist Leo Vygotsky during the first half of the 20th century (Leontiev et al., 1981). AT is a theory that seeks to understand who is doing what, why they are doing it and how the activity is done in a social context. Engeström (2001) describes AT as "a theory of object-driven activity". In the sense that, it provides scholars with a holistic framework and explanation for all the meaningful things people do to produce a change in practice. The basic unit of analysis in AT is the activity performed by humans (Kaptelinin et al., 2018; Ngoma et al., 2011). From the perspective of AT, an activity occurs when a "subject" uses certain tools to achieve an object. A subject could be an individual or an organization working together as a team with the aim of achieving an object (Kaptelinin et al., 2018). Objects are set goal (s) needed to be achieved by the subjects with the aid of certain tools. These Tools can be tangible or intangible. Examples of tangible tools are the hammer, spanner, mobile phone, or computer while intangible tools comprises ideas, signs or language (Ngoma et al., 2011). These concepts are interrelated and form part of an activity system. Hence, the nature of AT holds a relational ontology (Armstrong, 2011). The interrelationships among these concepts has the potentials of producing dynamic and conflicting perceptions through continuous interaction between and among these components in goal-directed activity (Wolff-Piggott \& Rivett, 2016a). The dynamic perceptions can be controlled by rules and a clear division of labor. Rules are the guiding principles that govern how and why a person acts within the activity system while. Division of labor defines specific roles and responsibilities of an individual subject in an activity system 
Activity Theory believes that the cultural-historical context of activity is key to understanding the dynamic nature and characteristics of an activity (Engeström, 2001; Wolff-Piggott \& Rivett, 2016b). This aspect of activity theory is an important when studies are done in the context of developing countries where activity systems are influenced by the societal and organizational context in which the activity exists (Karanasios \& Allen, 2013). Another fundamental concept in Activity Theory that is relevant to this study is contradiction. Contradictions are not simply problems or conflicts, they are "historically accumulating tensions among and between activity systems". Contradiction is an important principle in activity theory because it can lead to change and development (Brown, Allen et al., 2013). Contradictions are identified from empirical data in form of breakdowns and recurrent problems. They manifest in primary, secondary, tertiary and quaternary levels (Engeström, 2001). Primary contradictions are found within a component of an activity system. For example, conflicting rules may occur in situations where a physician may be forced, because of the economic strength of his patient, to prescribe cheaper and less effective drugs over the best (Riechert et al., 2016). Secondary contradictions manifest between components of an activity system. Secondary contradiction emerges when some elements like the division of labor or rules collide with new set of rules or roles. Tertiary contradictions are found "between the object of the central activity system and object of a more cultural advanced activity system". Quaternary contradictions appear in a network of activity systems between some components of the main activity system and the components of another activity system. Thus, activity systems change and develop by resolving their historically evolving contradictions (Engeström, Y, 1999). Furthermore, activity theory acknowledges contradictions as inevitable in the functioning of any activity system and identifies them as useful sources for developments in a system (Igira \& Aanestad, 2009).

\section{RESEARCH CONTEXT}

The setting of this research comprises selected PHCs in the Boko Haram affected areas of Adamawa State, Nigeria. Adamawa state is located in the North-Eastern (NE) region of Nigeria. The Boko Haram movement became militant in NE Nigeria in 2009. Consequently, Nigeria has been described as home to the deadliest armed conflict actors in Africa and was ranked as the third unsecured terrorized country in Africa (Ekhator-Mobayode \& Abebe Asfaw, 2019). Boko Haram is based on the Takfiri Islamic belief system, which opposes western education and views it as Haram (forbidden). Although a peaceful movement at inception, Boko Haram became violent in 2009 when the movement clashed with the Nigerian government because the government was against the ideology of Boko Haram (Badau \& Abdulrasheed, 2015). Boko Haram insurgency has displaced about 10,000 people in Borno and Adamawa states Most of the internally displaced people(IDPs) are from six Local Governments in Adamawa and Borno - Madagali, Gwoza, Michika, Gombi, Hong, Mubi North and Mubi south. This forceful movement has negative social and health impacts on the people of Adamawa state, especially children (Adamawa State Emergency Management Agency, 2014).

The research investigates the effectiveness of ALMANACH program in Adamawa State, Nigeria. ALMANACH was piloted in Adamawa state in December 2016 through a tripartite collaboration among the Adamawa State Primary Healthcare Development Agency (ADPHDA), the SWISS-TPH, and the International Committee of the Red Cross (ICRC). The ALMANACH is an electronic version of the integrated management of childhood illnesses (IMCI) running on mobile tablets. IMCI is a regulatory guideline that is in line with WHO's policy for improving the quality of child healthcare against the commonest child killer diseases in developing countries (malaria, measles, pneumonia, diarrhea, and malnutrition). Consequently, the ALMANACH was deployed as a clinical decision support tool to enhance the activities of the PHC workers during clinical consultations of children under the age of 5 by ensuring adherence to the IMCI guidelines. The ALMANACH provides a step-by-step process that guides the PHC worker to make accurate diagnosis and to administer correct dosage. It also suggests whether a patient needs referral to a higher care facility when necessary. The collaboration among the ADPHCDA, ICRC, and SWISS TPH is consistent with the ICRC Health Strategy, and complementary to the United Nations Sustainable Development Goals 3 (SDG3) (on ensuring good health) and SDG17 (pursuing partnerships to meet the goals). The long-time vision of the ALMANACH project is to scale up its application to all supported Primary Healthcare (PHC). This study seeks to provide additional insights that will help ALMANACH stakeholders realize their vision of a wide-scale-up. 


\section{RESEARCH METHODOLOGY}

A case study design was used to examine the use of ALMANACH in five local government areas (LGAs) namely; Yola South, Girei, Mubi-South, Mubi-North and Maiha. All the selected LGAs are located in armed-conflict (Boko Haram) parts of Adamawa State, Nigeria. Nineteen PHCs where ALMANACH has been deployed were studied, and the study was conducted mainly in children clinics. A conceptual understanding was developed by drawing insights from the Activity Theory in interpreting the data. A case study data collection procedure was used because the approach is suitable for investigating complex phenomena like mHealth (Yin, 2003).

The data collection took place through different but overlapping fieldwork phases. Data collection was done from March 2019 to July 2019 and from September 2019 to November, 2019. The ethnographic approach inspired the data collection methods adopted. The data collection procedure was guided by the activity theory principle of contradictions. Ethnography helps researchers to have a deep insight and describes how subjects utilize tools to accomplish an object in practice (Kaptelinin \& Nardi, 2018). We visited ten children clinics in different PHC centres in the state and observed clinical consultations. We collected the requisite data we need for the study and stored it in a database. We further reviewed clinical records (hospital cards) and the ALMANACH mobile tablet. We also participated in two steering committee meetings where all activities around ALMANACH were discussed with relevant stakeholders. A total of seventeen observation sessions were made across all selected PHCs with each session lasting between 1 to 3 hours. Interview was the main source of data collection techniques we deployed. A total of thirty-seven. Semi-structured interviews were conducted to have an understanding of work practices around ALMANACH. In some cases, focused group interviews were conducted for subset of health workers involved in the ALMANACH activity. This was augmented with one-on-one interviews where necessary. The interviews were conducted in Hausa and English languages. During observations and interviews, we interacted with three nurses, fifteen facility managers, nine CHEWs, and two laboratory technicians. However, because this type of interaction often leads to bias in sample selection, purposive sampling was used to select participants for the study. The participants were selected because they have met the inclusion criteria outlined for the conduct of the research. The criteria include being a member of ALMANACH team, experience in handling the ALMANACH system for at least six months and the ALMANACH must be deployed in an armed conflict zone of Adamawa State, Nigeria. Participants must also be 18 years of age and above before they eligible to participate in the study.

The data collection and analysis were guided by the Vygotsky's principles of contradictions which have been discussed in the previous section. Notes were taken during the focus group and interview sessions. In total, 22-page notes of qualitative data were collected, grouped, and analyzed using (Braun and Clarke (2006) principles of thematic analysis. This included careful and repeated readings of the data to get a summary of the main themes discussed by the participants. Next, a set of themes and relevant quotations from interviews were produced with relation to Vygotsky's principles of contradictions.

\section{ANALYSIS AND DISCUSSION}

Vygotsky's' principle of contradictions was used as the framework for the case study analysis. This was adopted in order to understand the challenges of using mHealth at PHCs in the selected study sites. These challenges manifested in the form of contradictions. Some of these contradictions have led to changes/ developments in the original activity system. Contradictions in ALMANACH system are then discussed.

\subsection{Contradictions within ALMANACH Systems (Primary Contradictions)}

The ALMANACH is deployed at the PHCs with the assumption that all clinical consultations of children under the age of 5 will be done using the ALMANACH. The present reality does not fit this assumption, as only one ALMANACH is assigned to each PHC and as it takes a long time to complete the process of a single clinical consultation (i.e. one child). The consequence to this is that facilities with a high turn-out of patients have developed other means of using the ALMANCAH whenever they have many patients awaiting consultation. 
The strategy the PHCs have employed is that while one health worker is using the ALMANACH to consult, another health worker records the vital signs of patients on a hard copy clinic card. In such scenario problem arises as some health workers sometimes forget to transfer all the data into the ALMANACH. Also, quality of care can be compromised thereby affecting the overall outcome of child healthcare, since some of the health workers work based on assumptions. For example, we witnessed discrepancies associated with data quality when we tried to compare entries made on the hard copy IMCI card and data recorded on the ALMANACH on some specified dates.

In some PHCs ALMANACH were powered off at the time there was electricity supply at the facilities. A health worker confessed to our team that they used the ALMANACH in their facility for only a month and since then they have been having problems whenever they try to use it again. Another staff said he feels the problem is that they do not understand how to use it. This highlights the lack of self-efficacy which was supported by the comment below:

"How can we even know how to use it properly when the person that was trained on how to use the ALMANACH is not a serious person and cannot use it herself?"

Another contradiction observed among the subjects (health workers) is related techno-phobia. Techno-phobia among health workers was confirmed at some facilities where some health workers stated that they no longer attend to children under the age of 5 since ALMANACH was deployed at their facility. This is because they would have to use the mobile tablet (ALMANACH) which they do not like. Therefore, they only consult adults and children above the age of 5 since they do not need any form of technology to attend to the medical needs of these populations. They said their decision not to consult children under the age of 5 was in agreement with all other staff of the facility.

The next sub-section presents contradictions that arise from the interaction of the ALMANACH activity system with other components within the activity systems.

\subsection{Secondary Contradictions}

Interactions among the sub-components of the ALMANACH produced some contradictions in the ALMANACH activity system, which could serve as potential threats to its scalability. The identified contradictions are elaborated in this sub-section.

There is no Internet network connectivity in some communities where the ALMANACH was deployed. This is especially the case at the Boko Haram host communities. As a result, facilities affected by this challenge devised a means through which they can overcome the problem. The facilities temporarily save all their information entered into the ALMANACH system and then travel to the nearest community with Internet connectivity to upload the data. This is done once in a week across all PHCs with similar problems. However, this development is further challenged by finance even though the sum of one thousand naira is included in their monthly budget. The money is to cover the purchase data bundle per month. A committed health worker narrated how their facility is trying hard to overcome this challenge in the following comment:

"This facility does not provide us with either money to buy a data bundle or the data bundle subscription. I have been using my money to buy data of about 200 naira weekly, and I have to also fuel my motor cycle to enable me to travel for about 2 kilometers to where I can be connected to internet service that will allow me to send our report. To be honest with you, I cannot promise you that I will continue to do this".

Delays in uploading of generated data at PHCs level could further have an impact on the disease surveillance system or other real-time analysis.

Other forms of secondary contradiction emanated from the rule and the division of labor components of the ALMANACH activity system. The rule guiding the use of ALMANACH states that only health worker is allowed to use the ALMANACH for medical consultations of children under the age of 5. But we found out that some laboratory technicians use the ALMANACH during clinical consultations of children. Such scenarios were seen at the Boko Haram host communities due to shortage of manpower. This contradiction led to a form of informal change in the division of labor in the facility. We have also observed that it has established a relationship between healthcare facilities. The laboratory technician had this to say:

"if not because of my presence here, this facility would have been under lock and key and the people here will have to travel some distance to receive any health care emergency needs". 
Also observed was the contradiction between tool and subject. This was made manifest during our interview sessions with the health workers where they mentioned that malaria was the most common disease in their communities and that it is one of the most dangerous killers of children under the age of 5. They also said that in their context, there are more than one different species of malaria and that, some malaria species cannot be seen when a blood sample is subjected to a particular type of malaria investigation. They have expressed their dissatisfaction with the ALMANACH arguing that ALMANACH recommends only a rapid diagnostic test (RDT) laboratory investigation to query malaria in a patient. A health worker at a different facility narrated his ordeal:

"A woman walked into this office crying and looking disappointed. She said to me that I had killed her daughter for her! She further said to me that, you should have told me that you don't know your job! My daughter died of severe malaria after you told me that there was nothing wrong with her".

There was also evidence of a contradiction between the clients and the subject. Some clients are of the view that subject (health workers) use the ALMANACH only because they are incompetent. Some health workers told us that some of their clients were bold to tell them the only reason they come to the clinics is that they have no other options. The facility had to organize orientation and sensitization sessions during antenatal, postnatal and immunization days to make the clients understand the reasons why the ALMANACH is used during clinical consultations of their children. These sessions somewhat changed the perceptions of many of the clients towards the use of ALMANACH. The clients also understood why they would have to go through all the processes involved before a prescription is made. This activity produced some visible positive outcomes.

\subsection{Quaternary Contradictions}

There were other protocols and programs for the management of children under the age of 5 across all the facilities visited. Other program protocols such as the standing order, IMCI chard, and save one million lives were deployed in the PHCs. A health worker said to us during an interview session with him that other protocols like the standing order covers many other illnesses which illnesses, which are not included on the ALMANACH. Again, even though, the ALMANACH is an electronic version of the IMCI, the IMCI chart is more detailed. As a result, some external evaluators and assessors score us high on other protocols in comparison to the ALMANACH system. The assessors often complain that the records we have on ALMANACH do not provide relevant information such as the client's address and phone number. They said they need this information to track the clients and to get feedback from them. For these reasons, we now ask for phone numbers of our clients and their addresses. Though we know there is no provision for it on the ALMANACH, we record it on the hard copy cards that we use. In this regard, a health worker said:

"In this clinic alone, we have been trained on the management of under 5 children by different intervention donors and each sponsor has a different approach to the management of children under the age of 5. The sponsors encourage us to use their program because as far as they are concerned, it is the best and so their supervision team always come around and we are always caught in-between one or more protocols".

\section{DISCUSSION AND CONCLUSION}

This study examined the challenges of mHealth in practice in the Boko Haram settings Adamawa State, NE of Nigeria. Findings from the study have uncovered several challenges in the form of contradictions that can impede the successful scale-up of ALMANACH in peculiar settings of Boko Haram. Hence, the findings of the study respond to concerns in the literature that uncovering contradictions are critical to achieving mHealth scale-up (Brown, Allen et al., 2013; Igira \& Aanestad, 2009).

The contradictions identified in the study were categorized into primary, secondary and quaternary contradictions. Following the Engeström (2009) principle of contradictions. The primary contradictions that were identified in the study are similar to others that were previously reported (e.g Ngoma, Faraja T. Igira, 2014; Kenny, Heavin, et al., 2017; Kenny, O'Connor, et al., 2017; Kruse et al., 2019) where mHealth is challenged by poorly trained health workers and poor data quality. Contrary to the findings of Kenny, O'Connor, et al (2017), a negative attitude towards mHealth was discovered in this study. Primary contradictions that were also discovered at the PHC level in this study include insufficient availability of mobile tablets (ALMANACH) and increased consulting time. Secondary contradictions were discovered from 
interactions between components of the ALMANACH activity system. The secondary contradictions are poor internet connection. This is mostly the case in the areas that have either been attacked by the Boko Haram or areas that are still experiencing attacks by Boko Haram. Poor funding policy at the PHC level, restrictions on certain laboratory investigations, shortage of healthcare workers especially in Boko Haram host communities, poor clients' knowledge and perception on mHealth were all secondary contradictions that were uncovered in the study. A quaternary contradiction was discovered from interactions among the ALMANACH system with other external systems. For instance, there were overlapping protocols for the management of children under the age of 5 from other intervention donors like UNICEF and the save one million lives intervention.

Insights from activity theory has guided the study to unearth the existence of some developments in ALMANACH practice (Igira \& Aanestad, 2009). Examples of such include: a form of a new division of labor, uploading of data to the main database has been rescheduled, an alternate source of funding has been created, rules limiting consultations at PHCs to only CHEWs have been changed to include laboratory technicians in the process of consultations using ALMANACH. Also, other malaria tests were done concurrently with RDT to satisfy contextual needs and CHEWs engaged some clients in conversations that changed their perception of ALMANACH.

Finally, in suggesting areas for future studies, the limitations of this study are acknowledged. The study was limited to a single case study. However, the study overcomes these limitations by the strength of qualitative data in providing deep insights (Abubakar \& Dasuki, 2018), into the experiences of health workers as it relates to the challenges of ALMANACH in practice. In conclusion, the study has made both practical and theoretical contributions. Activity theory helped in conceptualizing challenges as contradictions thereby exposing potential threats to achieving scale-up of ALMANACH to provide more insight into the pathways towards achieving scale-up of mHealth in armed conflict settings, our future study will examine the processes and outcomes of implementing ALMANACH.

\section{REFERENCES}

Abubakar, N. H., \& Dasuki, S. I. (2018). Empowerment in their hands: Use of WhatsApp by women in Nigeria. Gender, Technology and Development, 22(2), 164-183. https://doi.org/10.1080/09718524.2018.1509490

Armstrong, A. (2016). The Impact of Traditions and Traditional Birth Attendants on Maternal Mortality: A Case Study of Nyakayojo sub-County, Mbarara District, Uganda. 68.

Asi, Y. M., \& Williams, C. (2018). The role of digital health in making progress toward Sustainable Development Goal (SDG) 3 in conflict-affected populations. International Journal of Medical Informatics, 114, 114-120. https://doi.org/10.1016/j.ijmedinf.2017.11.003

Badau, K. M., \& Abdulrasheed, O. (2015). Management of Emergency Peace Education Programmes for Boko Haram Internally Displaced Persons in Yola Refugee Camp of Adamawa State Nigeria. Journal of Social Science Studies, 2(2), 36. https://doi.org/10.5296/jsss.v2i2.7138

Bilandzic, M., \& Venable, J. (n.d.). Towards Participatory Action Design Research: Adapting Action Research and Design Science Research Methods for Urban Informatics. 24.

Braun, V., \& Clarke, V. (2006). Using thematic analysis in psychology. Qualitative Research in Psychology, 3(2), 77-101. https://doi.org/10.1191/1478088706qp063oa

Brown, Allen, A., Karanasios, S., Leeds University, \& Norman, A. (2013). How Should Technology-Mediated Organizational Change Be Explained? A Comparison of the Contributions of Critical Realism and Activity Theory. MIS Quarterly, 37(3), 835-854. https://doi.org/10.25300/MISQ/2013/37.3.08

Chigona, W., Nyemba, M., \& Metfula, A. (2012). A review on mHealth research in developing countries. The Journal of Community Informatics, 9(2). http://ci-journal.net/index.php/ciej/article/view/941

Dehzad, F., Hilhorst, C., de Bie, C., \& Claassen, E. (2014). Adopting Health Apps, What's Hindering Doctors and Patients? Health, 06(16), 2204-2217. https://doi.org/10.4236/health.2014.616256

Devkota, B., \& van Teijlingen, E. R. (2010). Understanding effects of armed conflict on health outcomes: The case of Nepal. Conflict and Health, 4(1), 20. https://doi.org/10.1186/1752-1505-4-20

Ekhator-Mobayode, U. E., \& Abebe Asfaw, A. (2019). The child health effects of terrorism: Evidence from the Boko Haram Insurgency in Nigeria. Applied Economics, 51(6), 624-638. https://doi.org/10.1080/00036846.2018.1502871

Engeström, Y. (1999). Innovative learning in work teams: Analysing cycles of knowledge creation in practice. In Y. Engeström, R. Miettinen, \& R.-L. Punamäki (Eds.), Perspectives on Activity Theory. UK: Cambridge University Press. 
Engeström, Y. (2001). Expansive Learning at Work: Toward an activity theoretical reconceptualization. Journal of Education and Work, 14(1), 133-156. https://doi.org/10.1080/13639080020028747

Fanta, G. B., \& Pretorius, L. (2018). A Conceptual Framework for Sustainable Ehealth Implementation in Resource-Constrained Settings. South African Journal of Industrial Engineering, 29(3). https://doi.org/10.7166/29-32055

Igira, F., \& Aanestad, M. (2009). Living with Contradictions: Complementing Activity Theory with the Notion of "Installed Base" to Address the Historical Dimension of Transformation. Mind, Culture, and Activity, 16(3), $209-233$. https://doi.org/10.1080/10749030802546269

Kaptelinin, V., Kuutti, K., \& Bannon, L. (n.d.). Activity theory: Basic concepts and applications. 13.

Karanasios, S., \& Allen, D. (n.d.). Activity Theory in Information Systems Research. 5.

Kruse, C., Betancourt, J., Ortiz, S., Valdes Luna, S. M., Bamrah, I. K., \& Segovia, N. (2019). Barriers to the Use of Mobile Health in Improving Health Outcomes in Developing Countries: Systematic Review. Journal of Medical Internet Research, 21(10), e13263. https://doi.org/10.2196/13263

Leontiev, A. I., Mostinsky, I. L., Polonsky, V. S., Styrikovich, M. A., \& Chernika, I. M. (1981). Experimental investigation of the critical heat flux in horizontal channels with circumferentially variable heating. International Journal of Heat and Mass Transfer, 24(5), 821-828. https://doi.org/10.1016/S0017-9310(81)80005-1

Mangone, E. R., Agarwal, S., L’Engle, K., Lasway, C., Zan, T., van Beijma, H., Orkis, J., \& Karam, R. (2016). Sustainable Cost Models for mHealth at Scale: Modeling Program Data from m4RH Tanzania. PLOS ONE, 11(1), e0148011. https://doi.org/10.1371/journal.pone.0148011

Motamarri, S., Akter, S., Ray, P., \& Tseng, C.-L. (2014). Distinguishing "mHealth" from Other Healthcare Services in a Developing Country: A Study from the Service Quality Perspective. Communications of the Association for Information Systems, 34. https://doi.org/10.17705/1CAIS.03434

Ngoma, C., Chawani, M. S., \& Herstad, J. (2011). Adaptation of Mobile Application to Improve Flow of Birth Information from the Community to the District Level. In R. Popescu-Zeletin, I. A. Rai, K. Jonas, \& A. Villafiorita (Eds.), E-Infrastuctures and E-Services for Developing Countries (Vol. 64, pp. 79-92). Springer Berlin Heidelberg. https://doi.org/10.1007/978-3-642-23828-4_8

Riechert, J., Durst, C., \& Wickramasinghe, N. (2016). The Application of Activity Theory to Explain Collaborative Technology Use in Healthcare: The Case of a Chemotherapy Ordering System. 989-997. https://doi.org/10.1109/HICSS.2016.126

Sanner, T., Roland, L. K., \& Braa, K. (2012). From Pilot to Scale: Towards a Mhealth Typology for Low Resource Contexts. 13.

Schmied, V., Mills, A., Kruske, S., Kemp, L., Fowler, C., \& Homer, C. (2010). The nature and impact of collaboration and integrated service delivery for pregnant women, children and families: Collaboration in universal child and family health services. Journal of Clinical Nursing, 19(23-24), 3516-3526. https://doi.org/10.1111/j.1365-2702.2010.03321.x

Shidende, N. H. (2014). Challenges in Implementing Patient-centred Information Systems in Tanzania: An Activity Theory Perspective. The Electronic Journal of Information Systems in Developing Countries, 64(1), 1-20. https://doi.org/10.1002/j.1681-4835.2014.tb00455.x

Tomlinson, M., Rotheram-Borus, M. J., Swartz, L., \& Tsai, A. C. (2013). Scaling Up mHealth: Where Is the Evidence? PLoS Medicine, 10(2), e1001382. https://doi.org/10.1371/journal.pmed.1001382

WHO Global Observatory for eHealth, \& World Health Organization. (2011). MHealth: New horizons for health through mobile technologies. World Health Organization. http://www.who.int/goe/publications/goe_mhealth_web.pdf

Wolff-Piggott, B., \& Rivett, U. (2016a). An Activity Theory Approach to Affordance Actualization in mHealth : A Case of MOMCONNECT.

Woodward, A., Fyfe, M., Handuleh, J., Patel, P., Godman, B., Leather, A., \& Finlayson, A. (2014). Diffusion of e-health innovations in 'post-conflict' settings: A qualitative study on the personal experiences of health workers. 10.

Yin, R. K. (2003). Applications of Case Study Research. SAGE. 